Ann. Biol. anim. Bioch. Biophys, I965, 5 (I), 4I-5I.

\title{
INFLUENCE DES ANTIBIOTIQUES SUR LA RÉTENTION AZOTÉE CHEZ LE RAT EN CROISSANCE
}

\author{
A. RÉRAT, C. JOUANDET, J. LOUGNON \\ avec la collaboration techuique de Yvonne Danies, G. Rouzet, \\ Françoise Hovlier et M. Lecourtier \\ Station de Recherches sur l'Élevage des Porcs, \\ Centre national de Recherches zootechniques, Jouy-en-Josas (Seine-et-Oise)
}

\section{SOMMAIRE}

Le mécanisme de l'influence des antibiotiques sur la vitesse de croissance du Rat blanc et sur l'efficacité alimentaire du régime est étudié dans trois expériences où sont mises en œuvre des techniques d'alimentation diverses : alimentation mixte ad libitum, alimentation mixte restreinte pour obtenir une égalisation des gains de poids, alimentation séparée. Quels que soient le niveau et le mode d'alimentation, l'efficacité de la rétention azotée est améliorée par les antibiotiques. En revanche, les antibiotiques ne paraissent avoir aucune action directe sur le rendement de la rétention énergétique qui ne fait que subir le contrecoup des variations du niveau de consommation. Enfin, on peut déduire de ces faits que l'augmentation de consommation généralement constatée lors de l'antibiosupplémentation des régimes est due, au moins en partie, à l'amélioration de la rétention azotée par les antibiotiques.

L'addition d'antibiotiques aux aliments est devenue une pratique courante en élevage en raison de ses effets bénéfiques, notamment sur la vitesse de croissance des animaux et l'efficacité alimentaire. Si l'on est d'accord sur ce point, par contre leur mode d'action est sujet à controverse et de nombreuses hypothèses ont été émises (Françors, I962). Parmi ces hypothèses, certaines font état d'une action d'épargne sur les principes alimentaires grâce à une amélioration de l'utilisation digestive ou métabolique de la ration. C'est ainsi que l'épargne d'énergie a pu ètre mise en évidence par divers auteurs (NORDFEI,DT et KIMI,EN, I957; BRAUDE et Johnson, I953 ; Tiliman et Mac Vrcar, I956; Abraham et al., r958) alors que pour d'autres, il s'agissait avant tout d'une épargne d'azote (ForbEs, I954; Russo et al., I954; FÉVRIER et DUMON'T, I957; ZELTER et ChARLE'T-LÉRY, I960 ; THAYER et HELLER, I955).

Cependant, les méthodes qui ont permis d'aboutir à l'une ou l'autre de ces conclusions ne sont pas au-dessus de toute critique. Ainsi, les expériences ont été 
réalisées dans la plupart des cas en distribuant l'aliment à volonté, et les animaux témoins et antibio-supplémentés présentaient une vitesse de croissance très différente; or, ni dans le cas du métabolisme énergétique, ni dans celui du métabolisme azoté, ce ne sont les conditions requises pour comparer valablement des animaux en raison des variations du rapport entre la dépense de croissance et la dépense d'entretien. Par ailleurs, les quelques essais d'alimentation égalisée par paire selon la technique de MrTchel, (I930, I943) semblent prouver, aussi bien chez le Rat (Waisman et Boldt, I957) que chez la Volaille (SI.INGER et a1., I954) ou le Porc (WALLACE et al., I955), que la stimulation de croissance provoquée par les antibiotiques est en relation directe avec une élévation du niveau d'alimentation et s'expliquerait par cette seule augmentation. C'est pourquoi nous avons réalisé une série d'expétiences sur le Rat blanc en croissance dans le but :

- de déterminer si l'action d'épargne des antibiotiques sur le métabolisme azoté, déjà mise en évidence (RÉRAT, I96I) est doublée d'une action sur le métabolisme énergétique ;

- de préciser si l'action des antibiotiques passe en partie par le relais du niveau de consommation.

\section{MATÉRIEL ET MÉTHODES}

Deux séries d'expériences ont été entreprises, dans lesquelles sont utilisés, soit des régimes mixtes (expériences $\mathrm{A}, \mathrm{B}$ ) soit des régimes distribués selon la technique des repas séparés (expérience $\mathrm{C}$ ) déjà décrite par ailleurs (HENRY et RéRAT, I962).

Dans tous les cas, ces régimes sont administrés à des rats de souche Wistar C. F. pris au sevrage, placés dans des cages individuelles et répartis en lots, à raison de to animaux par lot, en fonction du poids et du gain de poids acquis durant une période préexpérimentale d'une semaine (MC KITTRICK, 1947), le régime étant à base de caséine (HENRY et RÉRAT, I962).

Le régime de base utilisé dans les expériences $\mathrm{A}$ et $\mathrm{B}$ est de composition très proche (tabl. $\mathrm{I}$ ) de celle des régimes déjà utilisés antérieurement (RÉRAT, I961), la farine blanche étant substituée aux céréales. Deux régimes de valeur biologique différente sont obtenus par addition à la farine blanche, soit de tourteau d'arachide $\left(R_{1}, R_{2}\right)$, soit d'un mélange arachide-levure $\left(R_{3}, R_{4}\right)$. Ces régimes

TABLEAU I

Composition des régimes utilisés

(Expériences $\mathrm{A}$ et $\mathrm{B}$ )

\begin{tabular}{|c|c|c|}
\hline & $\mathrm{R}_{1}$ & $\mathrm{R}_{\mathbf{3}}$ \\
\hline Farine blanche $\ldots \ldots \ldots \ldots \ldots \ldots \ldots \ldots$ & 73,0 & 68,4 \\
\hline Tourteau d'arachide ................ & 7,0 & 4,2 \\
\hline Levure .................... & - & 3,0 \\
\hline Amidon $\ldots \ldots \ldots \ldots \ldots \ldots \ldots \ldots \ldots \ldots \ldots \ldots$ & 9,0 & 13,4 \\
\hline Cellulose & 2,0 & 2,0 \\
\hline Huile d'arachide $\ldots \ldots \ldots \ldots \ldots \ldots \ldots \ldots \ldots$ & 5,0 & 5,0 \\
\hline Mélange salin $\left(^{1}\right) \ldots \ldots \ldots \ldots \ldots \ldots \ldots$ & 3,0 & 3,0 \\
\hline Mélangse vitaminique $\left.{ }^{1}\right) \ldots \ldots \ldots \ldots \ldots$ & 1,0 & 1,0 \\
\hline
\end{tabular}

(1) RÉRAT (1961). 
sont isoazotés (II p. 100 de matières azotées), isoénergétiques (4,25 Cal/g), antibio-supplémentés $\left(R_{2}, R_{4}\right)$ ou non $\left(R_{1}, R_{3}\right)$, et distribués à volonté (expérience $A$ ) ou en alimentation restreinte (expérience $B$ ) de telle façon que le gain de poids quotidien et par conséquent le besoin global d'entretien soient les mêmes pour tous les animaux (technique d'égalisation des gains de poids de Mitchell, I930). L'érythromycine, antibiotique extrait du bouillon de culture de Streptomyces erythreus, est utilisé dans ces deux expériences à raison de $50 \mathrm{mg} / \mathrm{kg}$ de régime.

Dans l'expérience C, conduite sur un principe différent, les rats reçoivent simultanément dans deux mangeoires séparées un régime protéiprive (tabl. 2) en libre consommation et un régime protidique (tabl. 2) distribué en quantité limitée (à raison de I,3 ou I,6 ou I,9 g de matières azotées par jour et par animal selon le lot) ; ce dernier régime est à base de tourteau d'arachide et contient ou non l'antibiotique (oxytétracycline : $0,72 \mathrm{mg} / \mathrm{j}$ par animal).

Dans tous les cas, les consommations individuelles de nourriture sont enregistrées quotidiennement et les animaux sont pesés deux fois par semaine. La consommation d'énergie est exprimée en

\section{TABLEAU 2}

Composition des régimes utilisés

(Expérience C)

\begin{tabular}{|c|c|c|}
\hline & $\begin{array}{l}\text { Régime } \\
\text { protéiprive }\end{array}$ & $\begin{array}{l}\text { Régime } \\
\text { protidique }\end{array}$ \\
\hline Sucre $\ldots \ldots \ldots \ldots \ldots \ldots \ldots \ldots \ldots \ldots$ & 860 & 118 \\
\hline Huile d'arachide $\ldots \ldots \ldots \ldots \ldots \ldots \ldots$ & 80 & 80 \\
\hline Cellulose $\ldots \ldots \ldots \ldots \ldots \ldots \ldots \ldots \ldots$ & 20 & 20 \\
\hline Mélange salin $(1) \ldots \ldots \ldots \ldots \ldots \ldots$ & 30 & 30 \\
\hline Mélange vitaminique ${ }^{(1)} \ldots \ldots \ldots \ldots \ldots \ldots$ & 10 & 10 \\
\hline Tourteau d'arachide..$\ldots \ldots \ldots \ldots \ldots$ & - & 692 \\
\hline $\begin{array}{l}\text { Prémélange à base de sucre contenant ou non } \\
\text { l'antibiotique } \ldots \ldots \ldots \ldots \ldots \ldots \ldots \ldots \ldots\end{array}$ & 一 & 50 \\
\hline $\begin{array}{l}\text { Analyse } \\
\text { Calories par } g \ldots \ldots \ldots \ldots \ldots \ldots \ldots \ldots \ldots \\
\text { Matières azotées }(p .100) \ldots \ldots \ldots \ldots \ldots\end{array}$ & $-^{4,45}$ & $\begin{array}{l}4,62 \\
39,3\end{array}$ \\
\hline
\end{tabular}

(1) RÉrat (1961).

Calories totales, d'après les coefficients d'Atwater et Bryant (I903). Les animaux sont sacrifiés à la fin des expériences dont la đurée est variable (expérience A : 45 jours ; expérience B : 49 jours ; expérience $\mathrm{C}: 28$ jours) et leurs carcasses sont analysées selon une technique déjà décrite (HENRY et RÉRAT, 1962; RÉRAT et al., 1964). Les calculs statistiques ont été effectués suivant la méthode d'analyse de variance (SNEDECOR, I956).

\section{RÉSULTATS}

\section{Régimes mixtes. Alimentation à volonté (expérience A)}

Les résultats généraux sont rapportés dans le tableau 3. La vitesse de croissance est augmentée par la présence d'érythromycine ou de levure dans les régimes, sans qu'il y ait interaction entre les deux effets. On constate parallèlement une consommation accrue de nourriture dans l'un ou l'autre cas. 
En ce qui concerne les coefficients rendant compte de l'efficacité alimentaire (C.U.P. calorique, C.U.P. azoté, C.E.P., C.E.E.), ils sont améliorés aussi bien par l'antibiotique que par la levure; il n'y a que dans le cas du coefficient d'utilisation pratique de l'azote qu'il existe une interaction entre l'effet antibiotique et l'effet levure, si bien que l'action des antibiotiques diminue lorsque la valeur biologique des protéines du régime est augmentée par la présence de la levure.

\section{TABLEAU 3}

\section{Expérience $A$. Résultats généraux}

(Durée : $45 \mathrm{j}$. Nombre d'animaux par lot : 12. Poids au départ : $76 \mathrm{~g}$ )

\begin{tabular}{|c|c|c|c|c|c|}
\hline Régime : Farine blanche $+\ldots$ & Arachide & $\begin{array}{c}\text { Arachide }+ \\
\text { Erythromycine }\end{array}$ & $\underset{\text { Levare }}{\text { Arachide }}+$ & $\begin{array}{c}\text { Arachide }+ \\
\text { Levure }+ \\
\text { Erythromycine }\end{array}$ & $\begin{array}{l}\text { Analyse } \\
\text { statistique } \\
\text { (l) }\end{array}$ \\
\hline \multicolumn{6}{|l|}{ Croissance el rétention } \\
\hline $\begin{array}{r}\text { Gain de poids quotidien moven } \\
\text { (g) } \ldots \ldots \ldots \ldots \ldots \ldots \ldots \ldots\end{array}$ & & & & & \\
\hline Ingéré énergétique (calories/j). & $\begin{array}{l}1,96 \\
51,3\end{array}$ & $\begin{array}{c}2,57 \\
58,8\end{array}$ & $\begin{array}{l}3,40 \\
61,9\end{array}$ & $\begin{array}{l}4,20 \\
72,6\end{array}$ & $L^{* *} A^{* *}$ \\
\hline Azote ingéré $(\mathrm{mg} / \mathrm{j}) \ldots \ldots \ldots \ldots$ & 269 & 295 & 324 & 382 & $L^{* *} A^{* *}$ \\
\hline $\begin{array}{l}\text { Coefficient d'efficacité énergéti- } \\
\text { que (C.E.E.) }\left(^{2}\right) \ldots \ldots \ldots \ldots \ldots \\
\text { Coefficient d'efficacité protidi- }\end{array}$ & 3,73 & 4,49 & 5,59 & 5,88 & $\mathrm{~L}^{* *} \mathrm{~A}^{* *}$ \\
\hline que (C.E.P.) $\left.{ }^{2}\right) \ldots \ldots \ldots \ldots$ & 1,16 & 1,38 & 1,70 & 1,77 & $\mathrm{~L}^{* *} \mathrm{~A}^{* *}$ \\
\hline $\begin{array}{l}\text { Énergie retenue (calories/j) .... } \\
\text { Coefficient d'utilisation prati- }\end{array}$ & 4,9 & 8,1 & 10,4 & 15,0 & $\mathrm{I}^{* *} \mathrm{~A}^{* *}$ \\
\hline que de l'énergie (C.U.P.E.) $\left.{ }^{2}\right)$ & 10,9 & 13,9 & 16,9 & 20,2 & $L * * A * *$ \\
\hline $\begin{array}{l}\text { Azote retenu }(\mathrm{mg} / \mathrm{j}) \ldots \ldots \ldots \ldots \\
\text { Coefficient d'utilisation prat- }\end{array}$ & 55 & 68 & 90 & 107 & $L^{* *} A^{* *}$ \\
\hline $\begin{array}{l}\text { Coefficient d'utilisation prati- } \\
\text { que de l'azote (C.Li.P.) }\left({ }^{2}\right) . . .\end{array}$ & 20,36 & 22,99 & 27,83 & 28,02 & $\mathrm{~L}^{* *} \mathrm{~A}^{* *} \mathrm{I}^{*}$ \\
\hline \multicolumn{6}{|l|}{ Composition corporelle } \\
\hline Poids au sacrifice $(\mathrm{g}) \ldots \ldots \ldots$ & 167,2 & $192,{ }^{\prime}$ & 232,5 & 267,6 & $L^{* *} A^{* *}$ \\
\hline Lipides p. $100 \ldots \ldots \ldots \ldots$. & 14,6 & 17,2 & 18,8 & 22,1 & $L^{* *} A^{* *}$ \\
\hline Protéines p. $100 \ldots \ldots \ldots$. & 17,5 & 17,0 & 16,9 & 16,4 & $\mathrm{~L}^{*} \mathrm{~A}^{*}$ \\
\hline Eau p. $100 \ldots \ldots \ldots \ldots \ldots$ & 64,4 & 62,5 & 61,2 & 58,5 & $L^{* *} A^{* *}$ \\
\hline Minéraux $p .100 \ldots \ldots \ldots \ldots$ & 3,5 & 3,3 & 3,1 & 3,0 & $L^{* * *} A^{* *}$ \\
\hline
\end{tabular}

(1) Analyse statistique. Seuil de signification : ${ }^{*}: 0,05 ; \quad * *: 0,01$.

$$
\mathrm{L} \text { : effet levure; } \mathrm{A} \text { : effet antibiotique; } \mathrm{I} \text { : interaction. }
$$

${ }^{(2)}$ C.E.E. : Gain de poids (g)/100 calories ingérées

C.E.P. : Gain de poids (g)/quantité de protéines ingérées (g)

C.U.P.N. : $\frac{\text { Azote retenu }}{\text { Azote ingéré }} \times 100$
C.U.P.E. : $\frac{\text { Energie retenue }}{\text { Energie ingérée }} \times 100$

En ce qui concerne la composition corporelle, elle est profondément modifiée par l'addition d'antibiotique ou de levure au régime ; le pourcentage de lipides dans la carcasse est augmenté dans des proportions importantes, cependant que diminuent 
les pourcentages de matières azotées, d'eau et de minéraux. Toutefois, il faut mentionner que le poids des animaux comparés est très différent, ce qui explique au moins les modifications de pourcentage de lipides et d'eau (RÉRAT et al., I964).

\section{Régimes mixtes. Alimentation restreinte (expérience $B$ )}

Les résultats généraux sont rapportés dans le tableau 4. Compte tenu de la restriction imposée de nourriture, les quatre lots d'animaux ont présenté une vitesse de croissance analogue, si bien que leur besoin d'entretien peut être considéré comme identique. Par rapport aux animaux recevant à volonté le régime à l'arachide $\left(R_{1}\right)$,

\section{TABLEAU 4}

Expérience B. Résultats généraux

(Durée : $49 \mathrm{j}$. Nombre d'animaux par lot : Io. Poids au départ : $66 \mathrm{~g}$ )

\begin{tabular}{|c|c|c|c|c|c|}
\hline Régime : Farine blanche + & Arachide & $\begin{array}{c}\text { Arachide + } \\
\text { Érythromycine }\end{array}$ & $\begin{array}{c}\text { Arachide } \\
\text { Levure }\end{array}$ & $\begin{array}{l}\text { Arachide } \div \\
\text { Levure } \div \\
\text { Erythromycine }\end{array}$ & $\begin{array}{c}\text { Analyse } \\
\text { statistique } \\
\text { (1) }\end{array}$ \\
\hline \multicolumn{6}{|l|}{ Croissance et rétention } \\
\hline Gain de poids moyen quotidien & 1.96 & 1,89 & 1,95 & 1.98 & N.S. \\
\hline Ingéré énergétique (calories/j). & 47,3 & 43,1 & 11,9 & 40,5 & $L^{* *} A^{* *} I^{* *}$ \\
\hline $\begin{array}{l}\text { Azote ingéré }(\mathrm{mg} / \mathrm{j}) \quad \ldots \ldots \ldots . \\
\text { Coefficient d'efficacité énergéti- }\end{array}$ & 248 & 224 & 219 & 215 & $L^{* *} A^{* *} I^{* *}$ \\
\hline 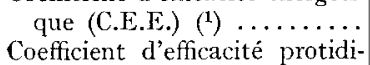 & 4,14 & 4,41 & 4,67 & 4,89 & $\mathrm{~L}^{* *} \mathrm{~A}^{* *}$ \\
\hline que (C.E.P.) ${ }^{(1)} \ldots \ldots \ldots$ & 1,26 & 1,35 & 1,42 & 1,47 & $\mathrm{~L}^{* *} \mathrm{~A}^{* * *}$ \\
\hline Énergie retenue (calories/j) ..... & 5,7 & 4,8 & 4,9 & 4,7 & N.S. \\
\hline $\begin{array}{l}\text { Coefficient d'utilisation prati- } \\
\left.\text { que de l'énergie }{ }^{(}\right) \ldots . . .\end{array}$ & 11,98 & 11,22 & 11,72 & 11,68 & N.S. \\
\hline Azote retenu $(\mathrm{mg} / \mathrm{j}) \ldots \ldots \ldots$ & $5 t$ & 56 & 61 & 63 & $\mathrm{~L}^{* *}$ \\
\hline $\begin{array}{l}\text { Coefficient d'utilisation prati- } \\
\text { que de l'azote }\left(^{1}\right) \ldots \ldots \ldots \ldots\end{array}$ & 21,56 & 25,00 & 27,99 & 29,33 & $L^{* *} A^{* *} I^{*}$ \\
\hline Composition corporelle & & & & & \\
\hline Poids au sacrifice $(g) \ldots$ & 168,0 & 159,0 & 161,3 & $16:, 7$ & N.S. \\
\hline Lipides p. $100 \ldots \ldots \ldots \ldots$ & $15,{ }_{4}$ & 12,5 & 12,1 & 11,2 & $\mathbf{L}^{* *} \mathrm{~A}^{* *}$ \\
\hline Protéines p. $100 \ldots \ldots$ & 17,4 & 18,3 & 19,0 & 19,2 & $L^{* *} A^{* *} I^{*}$ \\
\hline Eau p. $100 \ldots \ldots \ldots \ldots$ & 63,7 & 65,5 & 65,3 & 65,8 & $\mathrm{~A}^{*}$ \\
\hline Minéraux p. $100 \ldots \ldots$ & 3,6 & 3,7 & 3,6 & 3,7 & N.S. \\
\hline
\end{tabular}

(1) Voir tableau 3.

la restriction imposée aux autres animaux est de 1'ordre de 9 p. Ioo $\left(R_{2}\right)$, II,5 p. Ioo $\left(R_{3}\right)$ et I4 p. Ioo $\left(R_{4}\right)$, selon qu'ils reçoivent en outre l'antibiotique, la levure ou les deux substances; l'effet antibiotique est plus marqué en l'absence de levure qu'en sa présence (interaction très significative).

Si le rendement global de la ration (coefficient d'efficacité énergétique) est amélioré, il y a, en fait, dissociation entre les effets sur la rétention azotée et sur la 
rétention énergétique : l'efficacité de la première est significativement améliorée tant par la présence de levure que par celle d'antibiotique, l'amélioration due à l'antibiotique étant plus marquée lorsque la valeur biologique du régime est plus faible ; par contre, contrairement à ce qui se passe dans l'expérience $A$, l'efficacité de la rétention énergétique n'est en rien modifiée par l'addition de levure $\left(R_{3}\right)$ ou d'antibiotique $\left(R_{2}\right)$ au régime de base $\left(R_{1}\right)$. Bien que de poids égaux, les animaux présentent une composition corporelle différente; ils sont d'autant plus maigres que la restriction de nourriture a été plus sévère : c'est ainsi que le pourcentage de lipides est abaissé tandis que celui de protéines est augmenté lorsque le régime contient la levure ou l'antibiotique. Les modifications de composition corporelle dues à l'antibiotique sont plus marquées en l'absence de levure qu'en sa présence, ce qui va de pair avec la restriction imposée de nourriture.

\section{Régimes séparés. Apport azoté restreint (expérience $C$ )}

Les résultats généraux sont rapportés dans le tableau 5 .

TABIEAU 5

Expérience C. Résultats généraux

(Durée : 28 j. Nombre d'animaux par lot : Io. Poids au départ : $73 \mathrm{~g}$ )

\begin{tabular}{|c|c|c|c|c|c|c|c|}
\hline Lot $\ldots \ldots \ldots \ldots \ldots \ldots \ldots$ & $\mathrm{AO}$ & $\mathrm{AT}$ & $\mathrm{BO}$ & BT & $\mathrm{CO}$ & CT & \multirow{3}{*}{$\begin{array}{c}\text { Analyse } \\
\text { statistique } \\
\text { (I) }\end{array}$} \\
\hline Matières azotées $\mathrm{g} / \mathrm{j} \ldots \ldots \ldots \ldots$ & \multicolumn{2}{|c|}{1,3} & \multicolumn{2}{|c|}{1,6} & \multicolumn{2}{|c|}{1,9} & \\
\hline Oxytétracycline $\ldots \ldots \ldots \ldots \ldots$ & - & + & $\cdots$ & + & - & + & \\
\hline Croissance et rétention & & & & & & & \\
\hline Gain de poids quotidien $(g / j) \ldots \ldots$ & 1,24 & 1,38 & 1,72 & 1,86 & 2,03 & 2,21 & $A^{* *} N^{* *}$ \\
\hline Ingéré énergétique $(\mathrm{cal} / \mathrm{j}) \ldots \ldots \ldots$ & 38,9 & 40,6 & 43,0 & 45,5 & 45,1 & 48,4 & $A * * N^{* *}$ \\
\hline $\begin{array}{l}\text { Azote ingéré }(\mathrm{mg} / \mathrm{j}) \ldots \ldots \ldots \ldots \ldots \\
\text { Coefficient d'efficacité énergétique }\end{array}$ & 208 & 208 & 258 & 261 & 310 & 311 & $\mathrm{~N} * *$ \\
\hline $\begin{array}{r}\text { (C.E.E.) }{ }^{(2)} \ldots \ldots \ldots \ldots \ldots \ldots \ldots \\
\text { Coefficient d'efficacité protidique }\end{array}$ & 3,45 & 3,52 & 4,26 & $4,3 !$ & 4,73 & 4,87 & $\mathrm{~N}^{* *}$ \\
\hline (C.E.P.) $\left(^{2}\right) \quad \ldots \ldots \ldots \ldots \ldots$ & 1,02 & 1,11 & 1,12 & 1,21 & 1,08 & 1,21 & $A^{* *} N^{*}$ \\
\hline Énergie retenue (cal/j) $\ldots \ldots \ldots \ldots$ & 3,4 & 3,8 & 4,4 & 4,6 & 4,9 & 5,5 & $A^{* *} N^{* *}$ \\
\hline $\begin{array}{l}\text { Coefficient d'utilisation pratique de } \\
\text { l'énergie (C.U.P.E.) }(2) \ldots \ldots \ldots\end{array}$ & 8,9 & 9,1 & 10,4 & 10,3 & 11,0 & 11,4 & $\mathrm{~N}^{* * *}$ \\
\hline Azote retenu $(\mathrm{mg} / \mathrm{j}) \quad \ldots \ldots \ldots \ldots$ & 48 & 50 & 61 & 63 & 68 & 75 & $A^{* *} I^{*}$ \\
\hline $\begin{array}{l}\text { Coefficient d'utilisation pratique de } \\
\text { l'azote (C.U.P.N.) }\left(^{(2)} \ldots \ldots \ldots \ldots\right.\end{array}$ & 23,0 & 24,1 & 23,7 & 24,3 & 22,1 & $2^{\prime}, 3$ & $A^{* *} I^{*}$ \\
\hline Composition corporelle & & & & & & & \\
\hline Poids au sacrifice $(g) \ldots \ldots \ldots$ & 106,0 & 111,2 & 120,1 & 125,6 & 128,2 & 135,3 & $\mathrm{~A}^{* *} \mathrm{~N}^{* *}$ \\
\hline Lipides p. $100 \quad \ldots \ldots \ldots \ldots \ldots \ldots$ & 10,1 & 10,6 & 10,4 & 10,2 & 10,1 & 10,6 & N.S. \\
\hline Protéines p. $100 \ldots \ldots \ldots \ldots \ldots$ & 19,8 & 19,6 & 19,5 & 19,4 & 19,4 & 19,4 & N.S. \\
\hline Eau p. $100 \ldots \ldots \ldots \ldots \ldots \ldots$ & 66,4 & 65,9 & 64,5 & 67,0 & 67,0 & 66,5 & N.S. \\
\hline Minéraux p. $100 \ldots \ldots \ldots \ldots \ldots$ & 3,6 & 3,7 & 3,6 & 3,6 & 3,5 & 3,5 & N.S. \\
\hline
\end{tabular}

(1) Analyse statistique - seuil de signification : * 0,$05 ; \quad * *: 0,01$.

(2) Voir tableau 3 .

$\mathrm{A}$ : effet antibiotique ; $\mathrm{N}$ : effet niveau azoté ; I : interaction. 
Comme le prévoyait le schéma expérimental, les animaux ont ingéré la même quantité de matière azotée dans le lot témoin et le lot antibio-supplémenté à chacun des trois niveaux azotés. La vitesse de croissance est améliorée à la fois lorsque la quantité de matière azotée administrée aux animaux est augmentée et lorsqu'on ajoute un antibiotique dans le régime (amélioration significative de 9 à II p. Ioo). Les variations de l'ingéré énergétique vont dans le même sens : la supplémentation

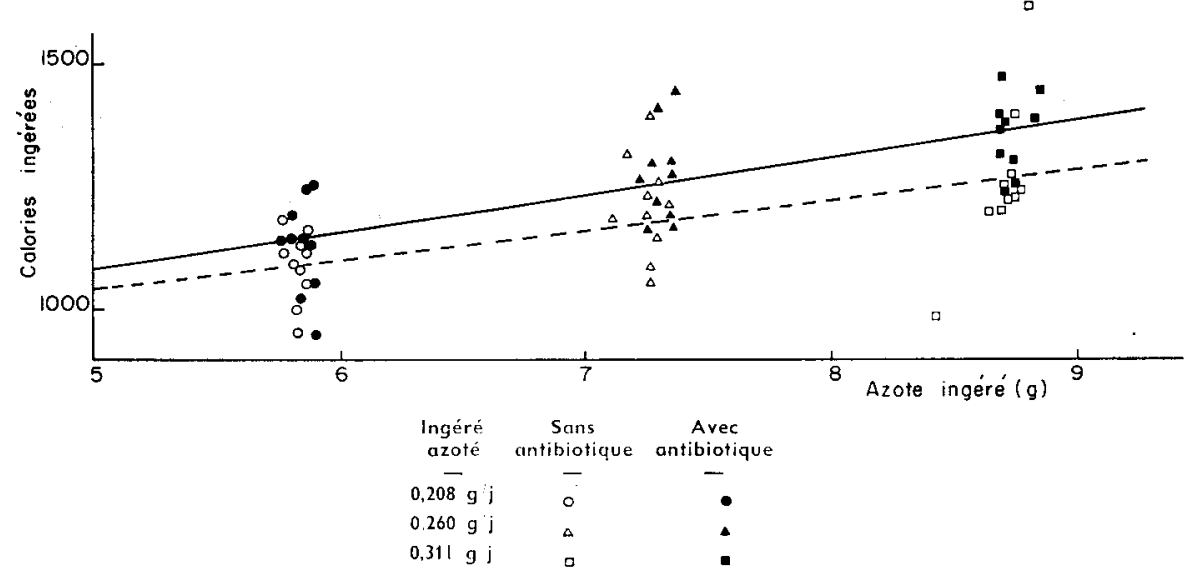

Fig. I. - Relation entre la consommaition d'énergie et la quantité d'azole ingéré :

Lots non supplémentés : $\quad \mathrm{Y}$ cal $=62 \mathrm{X}_{g}(\mathrm{x})+722 ;(r=0,55)$

Lots antibio-supplémentés : $\mathrm{Y}$ cal $=76 \mathrm{X}_{g}(\mathrm{x})+702 ;(r=0,74)$.

par l'antibiotique se traduit en particulier par une augmentation de la consommation de 6 à $7 \mathrm{p}$. Ioo. Cette augmentation est à peut près du même ordre à chaque niveau azoté ; il en résulte que, si l'on met en relation les calories ingérées et l'azote consommé on obtient, pour les animaux témoins et les animaux antibio-supplémentés, deux droites sensiblement parallèles et statistiquement distinctes dans les limites considérées (fig. I).

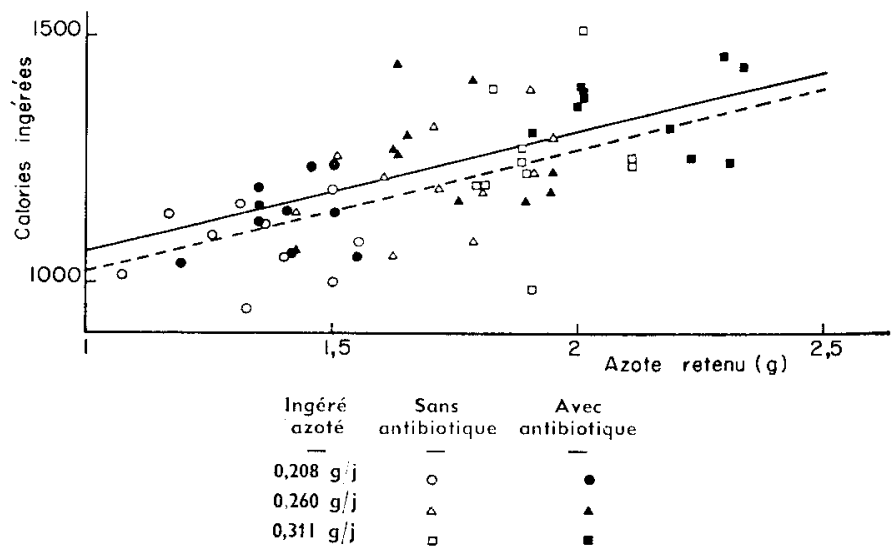

FIG. 2. - Relation entre la consommation d'énergie et la quantité d'azole reienu :

Lots non supplémentés : $\quad Y$ cal $=256 \mathrm{X}_{g}(\mathrm{~N})+760(\gamma=0,53)$

Lots antibio-supplémentés : $\mathrm{Y}$ cal $=243 \mathrm{X}_{g}(\mathrm{~N})+823(r=0,64)$ 
Le coefficient d'utilisation pratique de 1'azote, non modifié par le niveau azoté utilisé, est amélioré par 1'antibio-supplémentation. Autrement dit, pour une même quantité de matières azotées ingérées, la quantité d'azote retenue est plus forte en présence de l'antibiotique qu'en son absence. Compte tenu de l'augmentation parallèle de l'ingestion de calories, la relation entre les calories ingérées et l'azote retenu est la même pour les animaux antibio-supplémentés et pour les témoins (fig. 2). Contrairement à l'efficacité de la rétention azotée, celle de la rétention énergétique n'est pas modifiée par l'antibiotique, mais elle s'élève lorsque le niveau de consommation azoté est augmenté.

Enfin, bien que la vitesse de croissance des animaux n'ait pas été identique dans tous les lots, la composition corporelle des animaux ne varie sensiblement pas d'un lot à l'autre.

\section{DISCUSSION}

Sur le plan du métabolisme de l'azote, les résultats obtenus au cours des deux premières expériences ( $A$ et $B$ ) ne font que confirmer ceux d'une expérience antérieure (RÉRAT, I 96I). Comme dans cette expérience, les animaux ingérant ad libitum (expérience A) un régime hypoazoté contenant un antibiotique, l'érythromycine, ou un aliment azoté complémentaire tel que la levure, présentent une croissance et une rétention azotée accrues par rapport aux témoins. Cependant, on peut penser que ces faits sont liés au moins partiellement à la consommation accrue qui influe non seulement sur la quantité d'azote retenue, en valeur absolue, mais également sur le rendement azoté du régime puisqu'elle modifie le rapport entre dépense azotée de croissance et dépense azotée d'entretien. Les résultats de la deuxième expérience (B) permettent de lever cette indétermination. Dans ce cas, bien qu'on ait imposé aux animaux de tous les lots une vitesse de croissance identique, et que les quantités d'azote retenu soient pratiquement les mêmes pour un régime de valeur biologique donnée, l'efficacité de la rétention azotée est sensiblement améliorée d'une part par l'antibiotique, d'autre part par la levure. L'antibiotique valorise donc l'azote ingéré et son action est analogue à l'action de supplémentation d'un aliment protidique équilibré, la levure, vis-à-vis d'un régime hypoazoté à base de farine blanche et d'arachide. Enfin, l'expérience $C$ (alimentation énergétique ad libitum) confirme l'action bénéfique de l'antibiotique sur le rendement de la rétention azotée. On peut donc raisonnablement conclure que, dans nos conditions expérimentales, l'antibiotique provoque une épargne d'azote indépendante du niveau de consommation énergétique; il est toutefois impossible de préciser par ces expériences le niveau - digestif ou métabolique - oì elle intervient.

Par ailleurs, contrairement à ce qui se passe chez les animaux nourris ad libitum (expérience A), l'efficacité de la rétention énergétique n'est pas modifiée par la présence d'antibiotique ou de levure chez des animaux à croissance identique (expérience B). I1 semble done que les antiobitiques n'influent pas directement sur le métabolisme énergétique ; 1'action habituellement constatée passerait par le relais de la consommation. Compte tenu du fait que les régimes utilisés présentent un rapport Énergie/Protéines trop élevé, une consommation accrue ne peut se traduire que par 
un dépôt plus important de lipides : la part destinée à l'entretien devient, chez les animaux dont la consommation est augmentée, relativement faible par rapport à celle des dépôts alors qu'elle peut représenter la majeure partie de l'énergie ingérée lorsque la consommation est faible. On ne peut abandonner la question de l'énergie sans noter la discordance marquée entre le coefficient d'efficacité énergétique qui rend compte de l'utilisation apparente de l'énergie et le coefficient d'utilisation pratique de 1'énergie qui en traduit l'utilisation réelle.

En ce qui concerne le déterminisme de l'accroissement de la consommation chez les animaux recevant ad libitum un régime contenant l'antibiotique, l'expérience $\mathrm{C}$ permet d'en préciser certains aspects. Des travaux antérieurs (RÉRAT et al., I963; RÉRAT et HExRY, I963) réalisés à l'aide de la technique de l'alimentation séparée, ont en effet permis de trouver qu'il existe une relation constante entre la consommation spontanée d'énergie et l'intensité de la protéinogenèse imposée à l'animal par la quantité et la nature de l'apport protidique. Comme la rétention azotée est augmentée chez les animaux recevant des régimes antibio-supplémentés aussi bien en valeur absolue qu'en valeur relative, on peut penser qu'il s'agit là du facteur déterminant de l'augmentation de la consommation. De fait, la droite de régression liant l'ingestion d'énergie à l'azote retenu est pratiquement la même pour les animaux antibio-supplémentés et pour les témoins. Autrement dit, pour une rétention azotée donnée, l'énergie est ingérée en même quantité chez un animal antibio-supplémenté ou un témoin. Tout se passe donc comme si l'addition d'antibiotique agissait sur le niveau de consommation énergétique par l'intermédiaire du métabolisme azoté. Il n'en serait de même en alimentation mixte que lorsque le déséquilibre vis-à-vis des calories n'est pas trop prononcé dans le régime; dans ce cas, de la rétention accrue de matières azotées due aux antibiotiques, il peut résulter un excès de matières azotées utilisables par rapport aux calories; cet excès nécessite un apport accru d'énergie et entraîne une consommation supplémentaire de l'ensemble du régime. La nouvelle augmentation ainsi créée de l'apport et de la rétention azotés nécessite à nouveau un apport énergétique. L'ensemble de ces phénomènes aboutit finalement à un nouvel état d'équilibre entre azote et énergie. Il est probable que c'est ce qui s'est produit dans notre expérience $\mathrm{A}$. Cependant, cette explication n'est plus valable lorsque le déséquilibre entre azote et énergie dans le régime est plus marqué; les calories nécessaires à la rétention accrue d'azote due aux antibiotiques sont dès lors présentes dans le régime et une hyperconsommation ne devrait plus être nécessaire. Elle est toutefois signalée par de nombreux auteurs; la revue de VACHEL et FÉVRIER (I953) insiste particulièrement sur ce point.

En ce qui concerne la composition corporelle, rappelons tout d'abord qu'il est difficile de comparer valablement des animaux de poids très différents, les animaux les plus lourds étant les plus gras (RÉRAT et al., Ig64); précisons en outre que le nombre de données ne nous permet pas de calculer la régression des lipides ou des protéines tissulaires sur le poids vif. Compte tenu de ces réserves, on peut toutefois dire que, chez les animaux recevant des régimes mixtes (expériences A et B), les variations de la composition corporelle vont dans le même sens que celles de la consommation. Ainsi, dans l'expérience $A$, l'antibiotique ou la levure provoquent l'un et l'autre une augmentation de consommation. Il en résulte une augmentation des dépôts lipidiques et une diminution du pourcentage de protéines dans les tissus formés ; malgré la rétention azotée accrue, il subsisterait en définitive un excès de 
consommation d'énergie par rapport à l'azote disponible au niveau du métabolisme. A l'inverse, les animaux de l'expérience $B$ recevant l'antibiotique ou la levure dans le régime sont plus maigres que les témoins, ce qui est imputable à la réduction de consommation journalière qu'ils ont subie parallèlement à l'augmentation de la rétention azotée. Enfin, les animaux soumis à l'alimentation séparée (expérience C) présentent tous une composition corporelle voisine ; ce fait est conforme à nos résultats antérieurs (RÉRAT et HENRY, I963; HENRY et RÉRAT, I963) et s'explique par 1'ajustement en alimentation séparée du niveau de l'ingestion énergétique sur le niveau de rétention azotée.

Sur le plan méthodologique, la technique utilisée pour mesurer la rétention azotée s'est révélée à nouveau (RÉRAT, I96I) plus précise que la technique des bilans tournants utilisés dans des expériences antérieures (RÉRAT et al., I957) ; le bilan azoté garde néanmoins toute sa valeur pour décrire l'évolution đu métabolisme azoté : cette technique doit alors être appliquée pendant de longues périodes à des animaux dont la consommation a été égalisée par paire (MITCHELI, I930) et de nombreuses répétitions doivent être pratiquées.

En définitive, il apparaît de l'ensemble de ces expériences que les antibiotiques, utilisés à dose alimentaire, améliorent l'efficacité de la rétention azotée chez le Rat en croissance lorsque le régime est hypoazoté. Ce serait, pour une part, par ce relais que ces substances interviendraient sur le niveau de consommation et sur la vitesse de croissance des animaux.

\section{Reçu pour publication en novembre $\mathbf{1 9 6 4 .}$}

\section{SUMMARY}

\section{INFLUENCE OF ANTIBIOTICS ON RETENTION OF NITROGEN BY THE GROWING RAT}

A series of experiments was done with growing rats. The purpose was :

$\rightarrow$ to see whether the sparing action of antibiotics on metabolism of nitrogen, already demonstrated by RÉRAT (1961), is duplicated in an effect on metabolism of energy; of feed.

- to determine whether the effect of antibiotics operates partly through the effect on intake

In the first two experiments, each with 4 groups of ro weanling Wistar C. F. white rats, two diets of different biological value were given without or with antibiotic. They were given either to appetite or in amounts restricted to equalise growth of the different groups. In the third experiment, with 6 groups of ro white rats, the method of separate feeds was used, by which the protein feed of groundnut oilmeal was given at 3 levels, 1.3, I.6 or $1.9 \mathrm{~g}$ per day, and the protein-free feed was allowed to appetite.

From these experiments the following facts emerge :

Whatever the level or the method of feeding, antibiotic improves the efficiency of retention of nitrogen.

In contrast, these substances do not seem to have any direct effect on the efficiency of retention of energy, which only reflects the variation in the level of intake.

The increase in intake generally seen when an antibiotic supplement is given seems to be due, at least in part, to the improvement which the antibiotics cause in retention of nitrogen. 


\section{RÉFÉRENCES BIBIIOGRAPHIQUES}

Abraham J., Champigny O., JaCQuot R., r958. Influence de l'auréomycine sur les échanges respiratoires du Rat blanc. C. R. Acad. Sci., 246, 3520-3523.

Atwater W. O., Bryant A. P., i903. The chemical composition of american food materials. $U$. $S$. Dept. Agric., Bull., n० 28.

Braude R., Jolfnson B. C., r953. Effect of aureomycin on nitrogen and water metabolism in growing pigs. J. Nutr., 49, 505-512.

Février R., Dumont B. L., 1957. Auréomycine et lipogenèse chez le Porc. I. Ration pauvre en protéines. IV Congrès International de Nutrition, Paris. Résumés des communications, $78-79$.

Frasçors, 1962. Mode of action of Antibiotics on growth. World Review of Nutrition and Dietetics, 3 , 2I-64.

Forbes R. M., 1954. Studies on the influence of antibiotics and methionine on nitrogen utilization and basal metabolism of the growing male albino rat. $J . N u t r ., 53,275-288$.

HENRY Y., RÉRAT A., I 962 . Infuence de la qualité et de la quantité des matières azotées ingérées sur la consommation spontanée d'énergie chez le Rat en croissance. Ann. Biol. anim. Bioch. Biophys., 2, $267-276$.

Henry Y., RÉrat A., Ig63. Étude de l'ingestion spontanée d'éléments énergétiques et de protéines chez le Rat en croissance, par la méthode du libre choix. Ann. Biol. anim. Bioch. Biophys., 3, ro3-II7.

MAC KITTRICK D. S., 1947. The selection of chicks for growth experiments and the evaluation of growth. Growth, II, 89-99.

Mitchell H. H., r930. The paired feeding method : its value and limitation in livestock experimentation. Rec. Amer. Soc. Anim. Prod., $23 d$ ann. Meet., 63-73.

Mitchell H. H., ig43. Biological methods of value measuring the protein value of feeds. J. Anim. Sci., $2,263-277$.

Nordfeldt S., KIhLeN G., 1957. Studies of the influence of antibiotics upon the energy metabolism of young growing pigs. Kungl. Landtbrukskôgsk. Ann., 23, 457-477.

Rérat A., Abraham J., Champigny O., Jacquot R., 1957. Étude des effets comparés de l'érythromycine et de la levure sur la croissance et la rétention azotée du Ratiblanc. $C$. $R$. Acad. Sci., 245, 2 1 3-2 r6.

Rérat A., г96r. Les antibiotiques dans l'alimentation animale (Travaux de la Commission d'antibiotiques du C. N. E. R. N. A.) Ann. Nutr. Paris, 15, 132-I40.

Rérat A., Henry Y., JacQuot R., r963. Relation entre la consommation spontanée d'énergie et la rétention azotée chez le Rat en croissance. $C$. R. Acad. Sci., 256,1787-789.

Rérat A., Henry Y., I963. Étude de l'ajustement de la consommation spontanée d'énergie en fonction de l'apport azoté chez le Rat en croissance. Ann. Biol. anim. Bioch. Biophys., 3, 263-298.

Rérat A., Février C., Henry Y., Lougnon J., 1964. Évolution de läcomposition corporelle chez le Rat blanc en croissance. Ann. Biol. anim. Bioch. Biophys., 4, 35-47.

Russo J. M., Hanson L. E., Jezersi J. J., I954. The effect of aureomycin and arsanilic acid on nitrogen balance in pigs. J. Anim. Sci., 13, 998.

Slinger S. J., Hauser M. M., Pepper W. F., I954. The correlation between fced consumption and fecal flora in chicks. J. Nutr., 52, 75-88.

SNedecor G. W., r956. Statistical methods applied to experiments in_agriculture and biology. The Iowa State University Press, Ames, Iowa, $5^{\mathrm{e}}$ éd., pp. 534.

Thayer R. H., Heller V. G., I955. Antibiotics and nitrogen utilization in growing cockerels. Poultry Sci., 34, 97-102.

Tillman A. D., Mac VICAR R., 1956. The effect of chlortetracycline upon digestive of ration components retention of nitrogen and volume excreted by sheep with observations on rectal temperatures. $J$. Anim. Sci., 15, 21 I-2I7.

VACHel J. P., FÉvrier R., I953. Les antibiotiques dans l'alimentation_animale. Ann. Zootech., 1, I-87.

Waisman H. A., Boldt L. C., 1957. Influence of antibiotics and trytophan deficiency on growth in the rats. J. Nutr., 61, $457-464$.

Wallace H. D., Mc Kigney J. I., Pearson A. M., Cunha J. J., I955. The infiuence of chlortetracycline on the growth and carcass characteristics of swine fed restricted rations. J. Anim. Sci., 14, 1095I IO2.

Zelter S. Z., Charlet-Léry G., ig6o. Auréomycine et métabolismēazoté du Porc en croissance. Ve Congrès International sur la Nutrition, Washington; résumés, p.:2. 